\title{
Prospective Randomized Controlled Trial of Nimodipine as Add-On Therapy in the Treatment of Focal Refractory Epilepsy Patients: A Pilot Study
}

René Andrade Machado*, Edgar Bladimir Romero, Adriana María Goicoechea Astencio, Aisel Santos Santos, Vanessa Benjumea Cuartas and Marlene Maya López

Epilepsy Section, National Neurology and Neurosurgery Institute, Cuba

\begin{abstract}
Purpose: To prove whether the use of nimodipine as add-on therapy in focal refractory epilepsy patients could improve seizure control and quality of life.

Methods: This is a randomized, unblinded, controlled trial of nimodipine as add-on therapy in adult patients with focal refractory epilepsy. The trial consisted of baseline, maintenance and taper periods. Twenty-two patients with focal refractory epilepsy, which seizure frequency was greater than 5 per month, were randomized to one of the following dosage treatment regimens of nimodipine administered orally in four subdoses and according to body weight: arm A, $2 \mathrm{mg} / \mathrm{kg} / \mathrm{day} ;$ arm B, $3 \mathrm{mg} / \mathrm{kg} /$ day. The primary efficacy assessment, based on change in seizure frequency, was evaluated in two ways: the $50 \%$ responder rate and seizure freedom from baseline to maintenance periods; quality of life improvement was also taken into consideration. Assessment of adverse events (AEs) was included for safety evaluation.

Results: In the intention to treat population, $7.6 \%$ of patients were seizure free for 3 months. In 16/26 patients $(61.5 \%)$ seizure rate was reduced more than fifty percent. In the per protocol population 16/22 patients $(72.7 \%)$ seizure rate also diminished more than fifty percent. In those patients allocated to the arm $\mathrm{B}$, seizure frequency improved more than $50 \%$ in the first month of treatment. After three months of treatment, total quality of life improved significantly $(p<0.05)$. The most frequently reported adverse events were somnolence and headache.
\end{abstract}

Conclusion: Nimodipine improves quality of life and reduced seizure rate in patients with refractory focal epilepsy.

Keywords: Refractory focal epilepsy; Nimodipine; Add-on therapy; Randomized control trial; Drug resistance; Multidrug resistant protein; Calcium channel blocker

\section{Introduction}

Epilepsy is a common neurological disease, associated with significant morbidity, mortality, economic and social burden [1]. Despite of the availability of many antiepileptic drugs (AEDs), 20-40\% of all patients with epilepsy will continue to have frequent, disabling seizures, although maximum medical therapy is achieved [2]. Patients who have failed to respond to trials of up to two AEDs have a very low likelihood of responding to further medical therapy [3]. Although there are other options for treatment of medically refractory epilepsy including: surgical resection, callosotomy, multiple subpial transection, ketogenic diet, vagal nerve stimulator or transcranial magnetic stimulation and direct current brain stimulation, these interventions are expensive and not appropriated for all cases. That is why, for patients with refractory epilepsy, the situation has not improved significantly in the last few decades [4-6].

In the last years, there is increasing evidence that drug efflux transporters (namely $\mathrm{P}$-gp protein [P-glycoprotein] and MDRP [multidrug resistant protein] may play a role in drug-resistant epilepsy by limiting gastrointestinal absorption and brain access of antiepileptic drugs [7-13].

Calcium channel blockers are being used to reduce that drug efflux by interference with P-gp function in intestinal wall and in the apical membrane of brain capillary endothelial cells of the blood-brain barrier [11]. The most commonly calcium antagonist used to block the P-gp protein is Verapamil [14]. Nevertheless, from the clinical point of view, its pharmacokinetic interaction with most of the available AEDs limits its use for prospective clinical studies, where old antiepileptic medications such as Carbamazepine (CBZ) are usually employed [15$18]$.

By the other hand, nimodipine is a dihydropyridine calcium antagonist which has been shown to block the drug efflux such as verapamil but without clinically significant pharmacokinetic interactions with AEDs. Nimodipine has an adequate oral bioavailability and it is generally well tolerated, being hypotension the only adverse effect reported more frequently than with placebo treatment. Initially, oral treatment can be given at dosage of $60 \mathrm{mg}$ every 4 hours $[19,20]$.

Larkin et al. had previously investigated the usefulness of nimodipine in patients with focal epilepsy. The authors did not found any positive effect of this drug as adjunctive therapy (add-on) in the group of patients they analyzed. Nevertheless, the doses of nimodipine they employed were $30 \mathrm{mg}$ tds, $60 \mathrm{mg}$ tds, and $90 \mathrm{mg}$ tds, independently on body weight of those patients [21]. Taking into account this possible bias we have design the present study in which refractory focal epilepsy

*Corresponding author: René Andrade Machado, Epilepsy Section, National Neurology and Neurosurgery Institute, La Habana, Cuba, Tel: 8345504; E-mail: renemachado@infomed.sld.cu

Received February 25, 2013; Accepted May 18, 2013; Published May 30, 2013

Citation: Machado RA, Romero EB, Astencio AMG, Santos AS, Cuartas VB, et al. (2013) Prospective Randomized Controlled Trial of Nimodipine as Add-On Therapy in the Treatment of Focal Refractory Epilepsy Patients: A Pilot Study. J Neurol Neurophysiol 4: 151. doi:10.4172/2155-9562.1000151

Copyright: (c) 2013 Machado RA, et al. This is an open-access article distributed under the terms of the Creative Commons Attribution License, which permits unrestricted use, distribution, and reproduction in any medium, provided the original author and source are credited. 
Citation: Machado RA, Romero EB, Astencio AMG, Santos AS, Cuartas VB, et al. (2013) Prospective Randomized Controlled Trial of Nimodipine as Add-On Therapy in the Treatment of Focal Refractory Epilepsy Patients: A Pilot Study. J Neurol Neurophysiol 4: 151. doi:10.4172/21559562.1000151

patients received nimodipine -in association with previous antiepileptic medication-. Those patients have been randomized to two groups: Arm A, whom received $2 \mathrm{mg} / \mathrm{kg} /$ day and Arm B whom received 3 $\mathrm{mg} / \mathrm{kg} /$ day, to prove the efficacy and tolerability of nimodipine as addon therapy. We employed fractioned subdoses (four daily doses) and we also took into consideration body weight due to the possibility of obtaining more stable levels of the medication.

\section{Methods}

\section{Study design}

This is a randomized, unblinded, controlled trial of nimodipine as add-on therapy in patients with focal refractory epilepsy carried out in the National Institute of Neurology and Neurosurgery in Havana, Cuba. The study was approved by the ethic committee of the institution. Safety and data committees reviewed the protocol and enrollment annually.

\section{Patients}

\section{Inclusion and exclusion criteria:}

\section{a) Inclusion criteria}

1. Adult patients with focal symptomatic or cryptogenic refractory epilepsy aged between 18 and 55 years.

2. Patients without evidence of progressive structural lesion in the central nervous system (CNS) or progressive encephalopathy documented by clinical history or paraclinical investigation.

3. Patients with at least 5 partial-onset seizures per month, with no seizure-free interval longer than 21 days during the 8-weeks period before enrollment as well as during the 8 -weeks baseline period.

4. Patients with a stable dosage regimen of one to three AEDs, in the 4 weeks before enrollment and during baseline period.

5. To avoid the inclusion of patients with psychogenic seizures, all patients must have had a video-electroencephalography (EEG) study.

\section{b) Exclusion criteria}

1. Female patients were excluded from participation if they were pregnant, breast-feeding or of childbearing potential and not using approved methods of contraception.

2. Patients were excluded if they had participated in any other investigational trial within the last 2 months.

3. Patients were also excluded if they had a history of: chronic alcohol or drug abuse, any medical condition that might jeopardize patient's health or ability to participate in this trial; liver function test results of at least two times the upper limit of normal; creatinine clearance $<50 \mathrm{ml} / \mathrm{min}$; diastolic blood pressure $<50 \mathrm{~mm} \mathrm{Hg}$ or $>105 \mathrm{~mm} \mathrm{Hg}$ or heart rate $<50$ or $>110$ beats per minute after $3 \mathrm{~min}$ in a sitting position; heart rate by electrocardiography (ECG) $<50$ or $>110$ beats per minute; confirmed significant echocardiography abnormality; history of severe anaphylactic reaction or serious blood dyscrasias.

4. Patients with history of nonepileptic events, including psychogenic seizures.

5. Patients with previous history of: primary generalized seizures, status epilepticus in the last 12 months, any other clinically significant condition or recent chronic consumption of non-AED medications that might interfere with absorption, distribution, metabolism, or excretion of the drugs; treatment on a regular basis with one of the following group of medications that may influence the CNS function-neuroleptics, monoamine oxidase (MAO) inhibitors, barbiturates, except when taken as concomitant anticonvulsant treatment, or narcotic analgesics within 4 weeks before enrollment.

Refractoriness was considered when epilepsy failed to respond to two trials of well prescribed antiepileptic drugs accordingly to the epileptic syndrome the patient suffered. Treatment failure to an antiepileptic drug was considered when seizures were not controlled despite of the fact that the antiepileptic drug was increased up to maximum tolerated dose or a normal plasmatic level was documented. All patients had magnetic resonance imaging (MRI) or computed tomography (CT) scan exhibiting results consistent with focal epilepsy and without evidence of progressive structural lesion in the central nervous system (CNS) or progressive encephalopathy.

\section{Trial design}

- This trial consisted of baseline, maintenance, and taper periods. Enrolled patients entered after 8-weeks baseline period to obtain seizure frequency data, average of blood pressure and cardiac frequency and to evaluate the quality of life by applying QOLIE-31 questionnaire. Patients meeting the inclusion criteria were randomized according to aleatoric list created for the study and based on a predetermined computer-generated (pseudo-random number generator) schedule. Patients were randomized to one of the following dosage of nimodipine treatment regimens: arm A, $2 \mathrm{mg} / \mathrm{kg} /$ day or arm $\mathrm{B}, 3 \mathrm{mg} /$ $\mathrm{kg} /$ day. In all cases nimodipine was administered orally and divided in four subdoses daily. We employed fractioned subdoses and we also took into consideration body weight instead of using a fixed dose for group of patients, due to the possibility of obtaining more stable levels of the medication.

- Changes in dosage of AEDs during basal and follow-up periods were not allowed, except for those cases who experimented symptoms and/or signs of toxicity to which dose adjustment was recommended, but an increase of AEDs administered to them was forbidden in any circumstance.

\section{Follow-up}

After randomization, patients entered into a 12-weeks maintenance period. Each patient was instructed to visit our outpatient facility every month during the follow-up period or when they considered it was necessary. In the first contact, all patients received 4 questionnaires: 1.-Blood level questionnaire (to annotate the blood pressure they had every other day); 2. - Cardiac pulsations questionnaire (to annotate the cardiac frequency per minute every other day); 3.-Adverse health event questionnaire (to list any adverse event related to their health care) 4. - Seizure calendar questionnaire (to annotate the seizures calendar). This information was requested from patient's primary care physician and reviewed in each visit.

When side effects were reported, the physician evaluated whether it could be caused by antiepileptic medication. For this purpose, when available, plasma antiepileptic concentrations were measured. Then, doses were adjusted according to plasma levels. Otherwise, if plasma concentrations were not available, physicians were advised to reduce doses of AED until the side effect disappeared. When these options 
Citation: Machado RA, Romero EB, Astencio AMG, Santos AS, Cuartas VB, et al. (2013) Prospective Randomized Controlled Trial of Nimodipine as Add-On Therapy in the Treatment of Focal Refractory Epilepsy Patients: A Pilot Study. J Neurol Neurophysiol 4: 151. doi:10.4172/21559562.1000151

did not alleviate the symptoms, the patient was drop out of the study and nimodipine was tapered in one week. If during follow-up period, hypotension $(\mathrm{BP}<90 / 50 \mathrm{mmHg}$ ) or bradycardia (cardiac frequency per minute $<60$ ) were noticed, nimodipine was tapered rapidly.

\section{Efficacy assessment}

The primary efficacy assessment, based on change in seizure frequency, was evaluated in two ways: the $50 \%$ responder rate, from baseline to maintenance periods, defined as the proportion of patients experiencing $50 \%$ or greater reduction in seizure frequency, during 12 weeks from baseline to maintenance periods.

Secondary efficacy variables included the percent of change in seizure frequency during 12 weeks from baseline to maintenance period, number and proportion of patients achieving seizure-free status throughout the maintenance period in those who completed this phase and changes in the results obtained in QOLIE-31 questionnaire in baseline and maintenance period.

\section{Tolerability assessments}

Safety evaluation about nimodipine utilization included: assessment of adverse events (AEs) reported by patients or their caregivers and recorded in the adverse health event questionnaire or observed by the investigators, patient withdrawals due to AEs, changes in laboratory values (hematology, clinical chemistry, or urinalysis), vital signs, body weight measurements and physical and neurological examination findings.

\section{Statistical analysis}

Both, efficacy and safety analysis were performed on the Intentionto-Treat (ITT) population, which was defined as the population of patients included in the study, who took at least one dose of nimodipine. Efficacy was assessed as the percentage reduction in seizure frequency from baseline: the $100 \%$ responder rate (percentage of patients becoming seizure free), the $50 \%$ responder rate (i.e., percentage of patients with a reduction in seizure frequency of more than 50\%), and lack of response (patients who have improvement of their seizure rate of less than 50\%). All efficacy parameters were calculated for all seizures types combined (partial-onset or focal seizures, secondarily generalized seizures, convulsive or non convulsive ones and unclassified epileptic seizures). The retention rate, defined as the number of patients taking nimodipine at the end of the 12 -weeks treatment period divided by the total number of patients included in the study, was also used as a measure of efficacy.

Safety analysis focused on the nature, incidence and severity of adverse events. Kaplan Meier product-limit method was use to evaluate the time to reach fifty percent of responders. The differences between groups to reach fifty percent of responders were evaluate with Cox's F Test. Differences between two dependent variables were analyzed with Wilcoxon pair matched test. Differences between quantitative variables were calculated using T-Test. To evaluate differences in variability of blood pressure and heart rate were calculated with Friedman ANOVA and Kendall Coeff. of Concordance ANOVA Chi Squared. Only $p$ values less than 0.05 were considered significant.

\section{Results}

\section{General data of group of patients}

Demographic and clinical characteristics of patients included in the trial (ITT analysis set) are presented in (Table 1). Twenty two out of twenty six patients $(84.6 \%)$ completed the 12 -weeks of treatment; 10/13 (76.9\%) allocated to arm A and 12/13 (92.3\%) in the arm B (difference of proportion, $\mathrm{p}=0.27$ ). Three of them did not accept to continue taking the nimodipine without a clear reason and another also quit to continue with that drug, due to a severe headache. Retention rate was superior in arm B (92.7\% vs. $76.3 \%)$, but without statistical significance $(p=0.30)$. All patients were considered in our results. The average of days under treatment with nimodipine per patient (mean \pm SD) was $83.7 \pm 20.8$, (range 51-90 days) for those in arm $A$ and $76.5 \pm 27.9$ (range 49-90 days) for those in arm B, but the difference between both groups was non-statistically significant (Chart 1).

Compliance at all study: Compliance at all study visits was rated as excellent (e.g., $>90 \%$ medication intake based on pill counts) in at least $89 \%$ of subjects in both groups. Mean plasma concentrations of CBZ, determined in samples collected during follow-up, were significantly increased after nimodipine administration $(n=7)$ (Figure 1). Any sample had undetectable drug concentrations. Any patient was excluded from the analysis based on results of plasma drug concentration measurements.

Just in a few number of patients of those who were taking carbamazepine, measurement of that AED plasma levels was possible, because we had some technical problems at that time which did not allow test availability for all of them. In whom the test could be done, a significant increment was confirmed: plasma levels before treatment were $7.62 \pm 3.1 \mu \mathrm{g} / \mathrm{ml}$ range [4.2-12.1 $\mu \mathrm{g} / \mathrm{ml}]$ and after, $14.04 \pm 1.3 \mu \mathrm{g} / \mathrm{ml}$ [10.9-16.9 $\mu \mathrm{g} / \mathrm{ml}]$ ) (Figure 1).

\section{Effectiveness and drug exposure data}

Evaluation of efficacy: In the intention to treat (ITT) population, $7.7 \%(2 / 26)$ of patients were seizure free during the 3 months that lasted the study; those patients had been randomized to $3 \mathrm{mg} / \mathrm{kg} / \mathrm{daily}$ of nimodipine. In $61.5 \%$ (16/26 patients) seizure rate was reduced more than fifty percent. Seizure rate did not improve in around one third of cases.

In the per protocol (PP) population, $16.7 \%(2 / 22)$ of patients were

\begin{tabular}{|l|l|}
\hline Demographic and clinical characteristics & \multicolumn{1}{c|}{ mean \pm SD (range) } \\
\hline Age (years) & $39,9 \pm 11,7(21-64)$ \\
\hline Gender & $\mathrm{N}(\%)$ \\
\hline Female & $15(57,7)$ \\
\hline Male & $\mathrm{N}(\%)$ \\
\hline Type of epilepsy & $1(3,8)$ \\
\hline Neocortical temporal lobe Epilepsy & $19(73,1)$ \\
\hline Mesial temporal lobe epilepsy & $3(11,5)$ \\
\hline Frontal lobe epilepsy & $2(7,7)$ \\
\hline Parietal lobe epilepsy & $1(3,8)$ \\
\hline Occipital lobe epilepsy & mean \pm SD (range) \\
\hline AEDs employed (mg per day) & $1129 \pm 694,5(600-2000)$ \\
\hline Valproate & $1069 \pm 277,4(600-1600)$ \\
\hline Carbamazepine & $2 \pm 0,9(1-4)$ \\
\hline Clonazepam & $48 \pm 17,2(20-80)$ \\
\hline Clobazam & $250 \pm 50,7(200-300)$ \\
\hline Phenitoin & $361 \pm 111,2(200-600)$ \\
\hline Lamotrigine & $250 \pm 70,7(100-300)$ \\
\hline Topiramate & $10,55 \pm 3,4(4,26-12,3)$ \\
\hline Plasma level concentration before nimodipine $(\mu \mathrm{ml})$ & mean \pm SD (range) \\
\hline Carbamazepine & \\
\hline
\end{tabular}

Table 1: Demographic and clinical characteristics of patients included in the tria (ITT analysis set). 
Citation: Machado RA, Romero EB, Astencio AMG, Santos AS, Cuartas VB, et al. (2013) Prospective Randomized Controlled Trial of Nimodipine as Add-On Therapy in the Treatment of Focal Refractory Epilepsy Patients: A Pilot Study. J Neurol Neurophysiol 4: 151. doi:10.4172/21559562.1000151

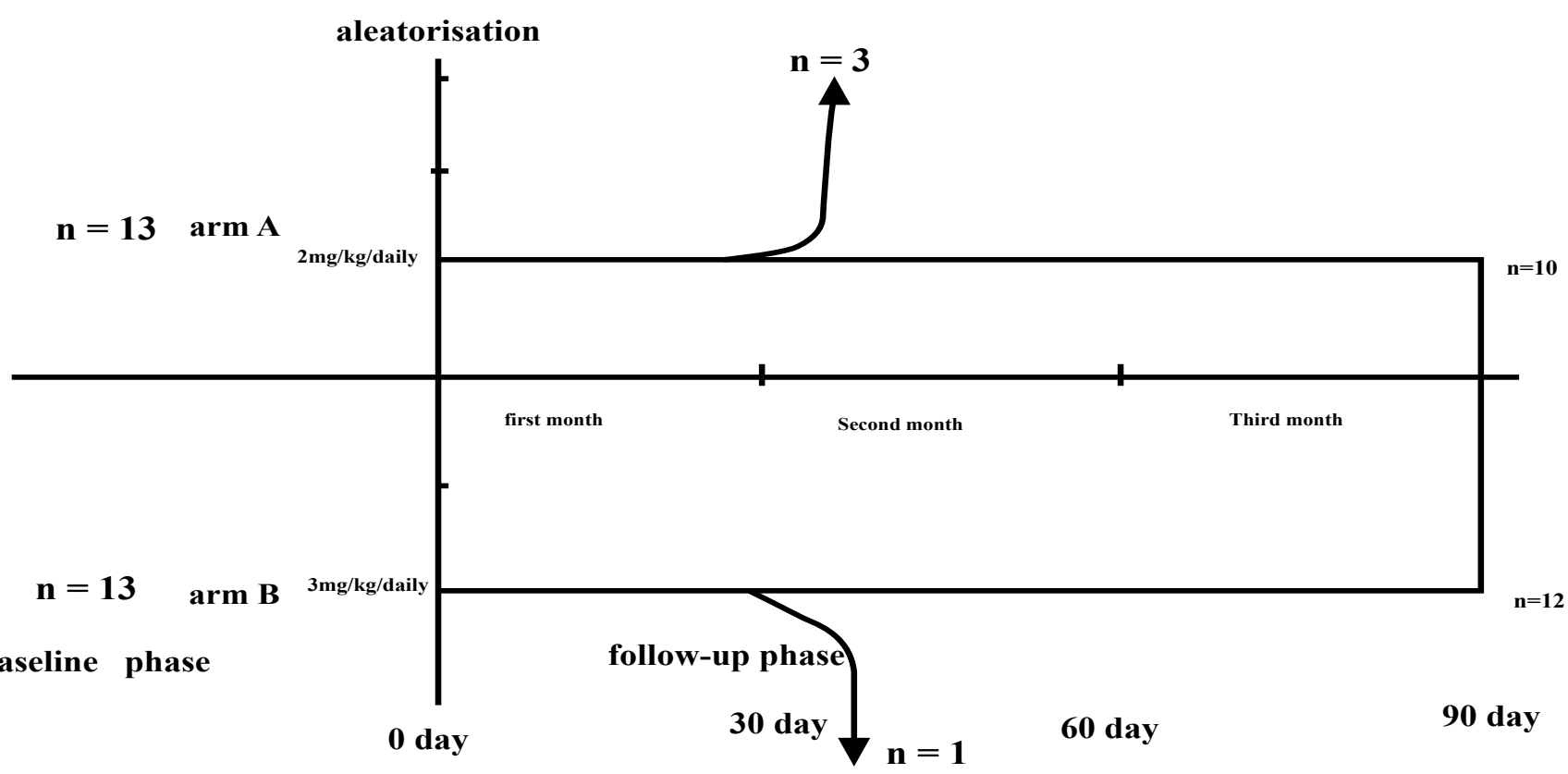

Chart 1: The black arrow signals the number of patients that dropped-out of the trial before one month of treatment. The average of total of days under treatment with nimodipine per patient according to the administered dose they were allocated to; T-Test (arm A vs. B) [83.7 \pm 20.8 (49-90) vs. 76.5 \pm 27.9 (51-90)], $p=0.35$. Retention rate by groups was 76.9 vs. $92.3(p=0.30)$

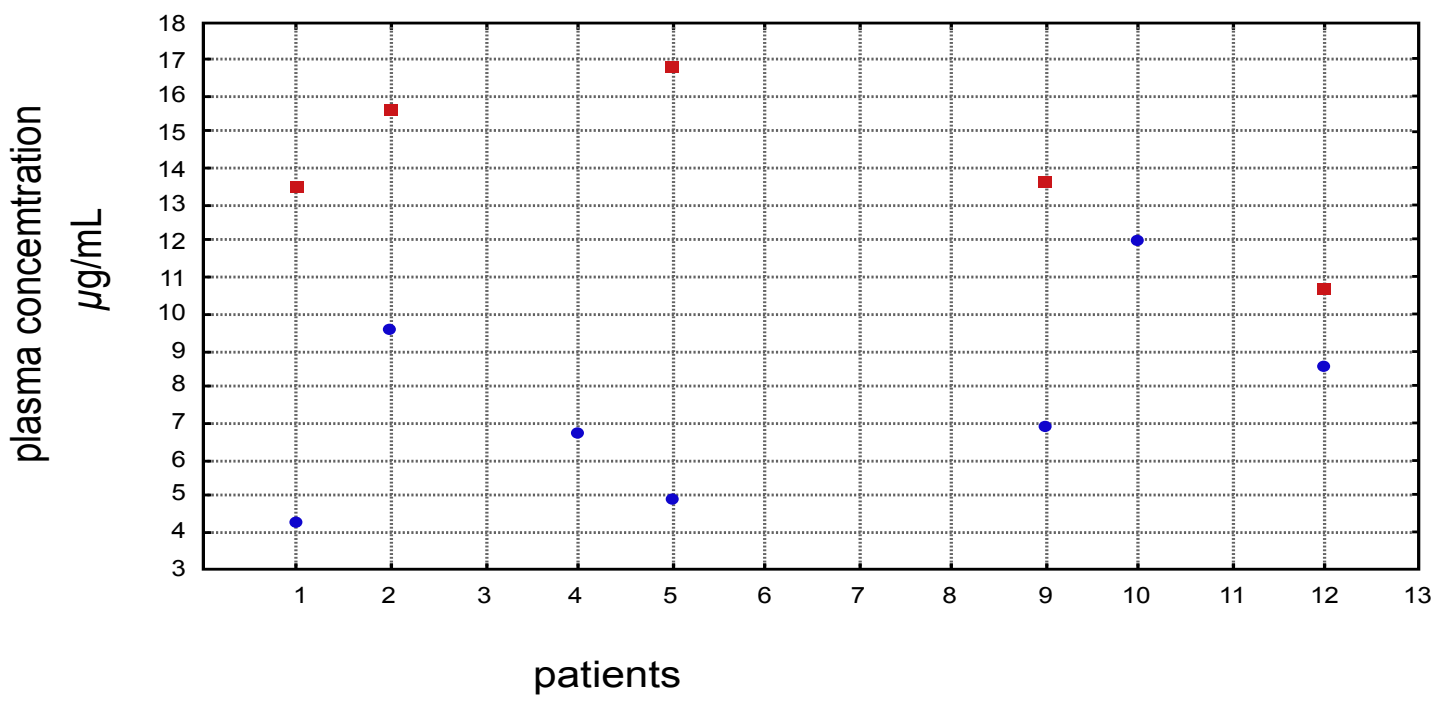

Plasma levels before treatment are market with blue circles and after treatment with red squares: before $(7.62 \pm 3.1 \mu \mathrm{g} / \mathrm{ml} \mathrm{range}[4.2-12.1 \mu \mathrm{g} / \mathrm{ml}]$ and after $14.04 \pm 1.3$ $\mu \mathrm{g} / \mathrm{ml}[10.9-16.9 \mu \mathrm{g} / \mathrm{ml}])$.

Figure 1: Mean plasma concentration of carbamazepine before and after 2 weeks of treatment with nimodipine.

seizure free during the 3 months of follow-up. In $72.7 \%(16 / 22)$ seizure rate was diminished more than fifty percent. Only in $13.6 \%$ of the patients who completed the study, seizure frequency did not improve. The effect of nimodipine was determined according to the dose of medication administered (Table 2).

Patients, in general, had a reduction of more than fifty percent in seizure rate in the second month of treatment (Figure 2A). Those patients allocated to the arm $\mathrm{B}$, reached a $50 \%$ responder rate in the first month of treatment, whereas in those who corresponded to group A this goal was reached in the third month after treatment. This difference reaches statistical significance $(\mathrm{p}<0.05)$ (Figure 2B).

The total QOLIE-31 score and all quality of life subscales, improved significantly after nimodipine treatment $(\mathrm{p}<0.05)$, except domains related to cognitive effects and side effects of medications. Although the differences between score for the last two mentioned quality of life subscales did not reach statistical differences $(p>0.05)$ they improved 
Citation: Machado RA, Romero EB, Astencio AMG, Santos AS, Cuartas VB, et al. (2013) Prospective Randomized Controlled Trial of Nimodipine as Add-On Therapy in the Treatment of Focal Refractory Epilepsy Patients: A Pilot Study. J Neurol Neurophysiol 4: 151. doi:10.4172/21559562.1000151

Page 5 of 8

\begin{tabular}{|c|c|c|c|}
\hline Nimodipine dose & Seizure freedom n (\%) & More than $50 \%$ of seizure rate reduction but not seizure free * & Lack of efficacy * $\mathrm{n}(\%)$ \\
\hline \multicolumn{4}{|c|}{ Intention to treat analysis } \\
\hline Arm B n=13 (3 mg/kg/day) & $2(15.3)$ & $8(61.5)$ & $2(15.3)$ \\
\hline Arm A n=13 (2 mg/kg/day) & ---------- & $8(61.5)$ & $5(38.4)$ \\
\hline Total $=26$ & $2(7.69)$ & $16(61.5)$ & $7(26.9)$ \\
\hline \multicolumn{4}{|c|}{ Per protocol analysis } \\
\hline Arm B n=12 (3 mg/kg/day) & $2(16.7)$ & $9(75)$ & $1(8.3)$ \\
\hline Arm A n=10 (2 mg/kg/day) & ---------- & $7(70)$ & $2(20)$ \\
\hline Total $=22$ & $2(9.1)$ & $16(72.7)$ & $3(13.6)$ \\
\hline
\end{tabular}

*differences between proportion of patients with more than $50 \%$ of seizure free, lack of efficacy and seizure freedom p>0.05 (Chi squared test).

Table 2: Rate of response according to nimodipine dosage employed.
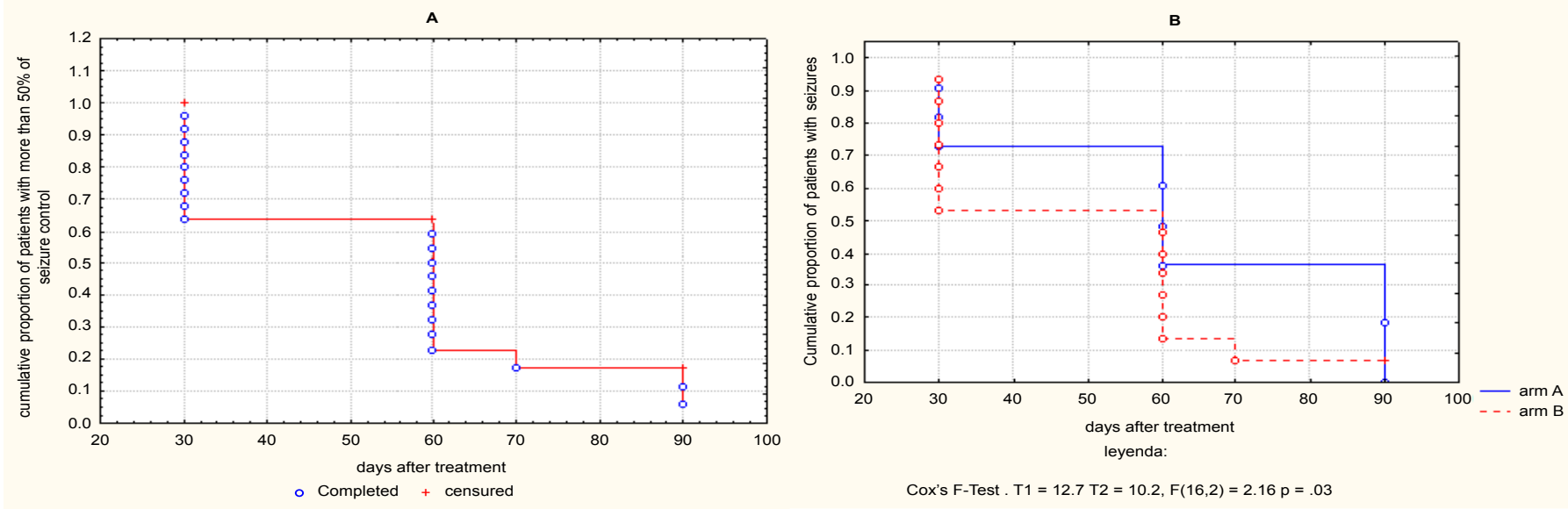

A-blue circles: completed treatment and red cross: censured treatment B-blue lines: Arm A and red lines: Arm B

Figure 2: Efficacy of nimodipine treatment in association with AEDS in patients with focal refractory epilepsy. A: Time to reach $50 \%$ of seizures reduction in the intention-to-treat population (cumulative proportion of patients with seizures in both groups of study). B: (cumulative proportion of patients with seizures according groups of randomization).

after treatment with nimodipine (Cognitive effects $67.9 \pm 22.5$ vs. 76.3 \pm 19 and Drug side effects $41 \pm 19.6$ vs. $45.9 \pm 17.6$ ) (Table 3 ).

Tolerability: Five out of twenty-six cases -which corresponded to $19.2 \%$ - experienced at least one adverse event, with $7.7 \%$ of them considered to be related to nimodipine usage. In $3 / 26(11.5 \%)$ the reported adverse event led to antiepileptic dose reduction; only one patient discontinued nimodipine treatment for this reason. This patient had been randomized to nimodipine $3 \mathrm{mg} / \mathrm{kg} / \mathrm{daily}$ and he complained of intense headache. The percentage of side effects was not different between the groups of study. A little increase in percentage of side effects, was seen in the group of nimodipine at dose of $3 \mathrm{mg} / \mathrm{kg} /$ daily $(15.4 \%$ vs. $23.7 \%$ ), but this difference did not reach statistical significance. The total QOLIE-31 improved in both groups of randomization, and this increment did not depend on the dose of nimodipine used (Table 4).

The most frequently reported adverse events ( $>5 \%$ of the patients), were related to the nervous system (somnolence and headache). No one reported a serious adverse event that was considered to be related to nimodipine treatment. Headaches (7.7\%) and somnolence (11.5\%) were the most frequently reported adverse events (Table 4). Dizziness, somnolence and diplopia improved after changing carbamazepine or phenytoin doses (Table 5).

Significant changes in heart rate, systolic blood pressure and diastolic blood pressure, were not found during follow-up (Table 6). Any patient needed to withdraw treatment due to cardiovascular side effects.

\section{Discussion}

In the clinical set point, approximately one third of patients with epilepsy do not respond to antiepileptic drugs (AEDs) and it has been hypothesized that this is due to over-expression of the multidrug transporter. Over-expression of P-glycoprotein (P-gp) has been reported to play an important role in refractoriness to AEDs [22,23]. This protein functions as a transmembrane efflux pump, by moving drugs from the intracellular to the extracellular domain. It is a product of the ATP-binding cassette subfamily B member 1 (ABCB1) gene [24]. It has been hypothesized that genetic variation may be involved in resistance to treatment [25]. Lannetti et al. reported a patient who was treated with verapamil for prolonged and refractory status epilepticus with magnificent results [26]. Taking into account these evidences we conducted the present study.

Analysis of the results of the current trial showed that nimodipine improved seizure rate and quality of life without significant side effects when added to a stable regimen of one or two anti-epileptic drugs. Frequency of partial seizures and of all seizure types combined was reduced more than $50 \%$. Seizure freedom, which is the ultimate goal of treatment, was achieved in only $2(7.7 \%)$ cases of the studied population (intention to treat analysis) (Table 2). Nevertheless, more than half of the patients $(61.5 \%)$ were $50 \%$ responders (i.e., reported a reduction in seizure frequency of more than $50 \%)$. Some differences favors the indication of $3 \mathrm{mg} / \mathrm{kg} /$ daily of nimodipine: in the per protocol analysis, at this dose, approximately $80 \%$ of patients reduced 
Citation: Machado RA, Romero EB, Astencio AMG, Santos AS, Cuartas VB, et al. (2013) Prospective Randomized Controlled Trial of Nimodipine as Add-On Therapy in the Treatment of Focal Refractory Epilepsy Patients: A Pilot Study. J Neurol Neurophysiol 4: 151. doi:10.4172/21559562.1000151

\begin{tabular}{|c|c|c|}
\hline \multirow{2}{*}{ QOLIE-31 } & Before treatment & After treatment \\
\cline { 2 - 3 } & Mean \pm SD (range) & Mean \pm SD (range) \\
\hline Total quality of life & $51.3 \pm(29.4-89.8)$ & $62.6 \pm 15.4(32.586)^{* *}$ \\
\hline Seizure worry & $55.2 \pm(0-100)$ & $73.4 \pm 18.2(18-100)^{* * *}$ \\
\hline Emotional well being & $48.6 \pm 21.3(12-100)$ & $62.1 \pm 20.6(20-89)^{* * *}$ \\
\hline Energy/fatigability & $49.1 \pm 21.5(20-95)$ & $61.6 \pm 19.4(30-95)^{* * *}$ \\
\hline Drug side effects & $41 \pm 19.6(6.6-74)$ & $45.9 \pm 17.6(17.5-75)^{*}$ \\
\hline Cognitive effects & $67.9 \pm 22.5(16.6-100)$ & $76.3 \pm 19(47.2-100)^{*}$ \\
\hline Social functioning & $53.7 \pm 27.4(14-95)$ & $71.3 \pm 25.5(19-100)^{* * *}$ \\
\hline
\end{tabular}

Table 3: Quality of life before and after treatment with nimodipine (ITT analysis).

\begin{tabular}{|c|c|c|c|}
\hline Tolerability & $\begin{array}{c}2 \mathrm{mg} / \mathrm{kg} \text { (before/ } \\
\text { after) }\end{array}$ & $\begin{array}{c}3 \mathrm{mg} / \mathrm{kg} \\
\text { (before/after) }\end{array}$ & $\begin{array}{c}\text { Statistic } \\
\text { (p value) }\end{array}$ \\
\hline $\begin{array}{c}\text { Dropped-out because side } \\
\text { effects of medication }\end{array}$ & $0(0)$ & $1(7.7)$ & 0.93 \\
\hline$\%$ of side effects & $2(15.4)$ & $3(23.7)$ & 0,37 \\
\hline Total QOLIE-31 & $(51,2) /(62,3)$ & $(52,4) /(61,9)$ & 0,78 \\
\hline
\end{tabular}

Table 4: Tolerability and changes in QOLIE-31 according to nimodipine dose.

\begin{tabular}{|c|c|}
\hline Side effects & $\mathrm{n}(\%)$ \\
\hline Headache & $2(7,6)$ \\
\hline Anorexia & $1(3,8)$ \\
\hline Diplopia \pm & $1(3,8)$ \\
\hline Somnolence \pm & $3(11,5)$ \\
\hline Nausea & $1(3,8)$ \\
\hline Dizziness \pm & $1(3,8)$ \\
\hline Weight increment & $1(3,8)$ \\
\hline Fatigue & $1(3,8)$ \\
\hline
\end{tabular}

Side effects improving after diminishing of AEDs doses appear market with \pm . Only carbamazepine and Phenytoin were diminished when necessary.

Table 5: Principal side effects reported during treatment (intention to treat analysis).

seizure rate by more than $50 \%$, compared to $66,6 \%$ in the group of $2 \mathrm{mg} / \mathrm{kg} /$ daily; individuals who remained seizure free belonged to the group that received $3 \mathrm{mg} / \mathrm{kg} /$ daily. This group also reached reduction of fifty percent of seizures within the first month of treatment, whereas in the other group, this goal was reached after two months of treatment; difference which reaches statistical significance. Although there was a small increase in the percentage of side effects with higher doses of nimodipine, this was not statistically significant (Table 4 ).

Contrary to our results are those published by Larkin et al. [21]. In their study the authors found that in patients treated with nimodipine for 12 weeks, the median values of seizures rate (placebo vs. nimodipine) did not vary for total (17 vs. 18 ), partial (14 vs. 18) and generalized tonic-clonic seizures ( 2 vs. 5$)$ or seizure days (13 vs. 13) either. Antiepileptic drug levels were not affected by nimodipine treatment. By the way, our results mirror in some aspects what Larkin's et al. study found. In both investigations the retention rate was similar because the authors reported 2 non-compliant patients too, although the withdrawal by side effects was superior in their study [21] (2 vs. 1 in our research).

We have to mention some differences between Larkin's trial and our investigation. The mains differences are: the use of placebo and a double blind design in the previous study, the lower nimodipine doses chosen by the authors and the specific populations selected. The first aspect favored methologically to Larkin's research as its design avoids the placebo effects of medication. Nevertheless, the second difference could largely affect the results and the interpretation because many of AEDs employed in their research were enzyme-inducing drugs, which may cause low circulating nimodipine concentrations avoiding its action in the Pgp system and thus, the possible lost in the effectiveness. Furthermore, our group of patients had a higher seizure frequency per month, which allowed us to evaluate response rate easier. The problem of possible methodological bias that explains the differences between the investigation and our trial are discussed in the following paragraphs [21].

Larkin's JG et al. research is a double blind, prospective, controlled design but possible biased because the use of nimodipine did not take into account the body weight to calculate or recommend specific doses for patients [21]. On the other hand, our trial is an open label study. In this type of trials, the placebo effect cannot be excluded but minimized, because we randomized patients into two groups which received different doses of nimodipine. This design, try to find the dose-dependent effect. We know it could be misleading because the effective dose of nimodipine in epilepsy patients is uncertain. However, if the use of a placebo is impractical, a multiple fixed-dose study without a placebo may be an acceptable option. This approach involves comparison of responses to a range of doses of the test drug (ex., nimodipine). If significant statistical differences are observed between treatment groups, such an outcome is interpreted as evidence of a drug's efficacy. This can be an economic approach to drug development because it generates valuable dose-response data in addition to supporting drug efficacy. However, it carries the potential risk of a false negative error if the wrong doses are selected because of uncertainty about the therapeutic dose range of a particular drug. Choosing inappropriate doses to be evaluated can lead to failure, especially if the lowest dose selected has some therapeutic benefit.

Meyer et al realized an investigation in which prospective and double blinded patterns were used with a follow up period of 12 months, no effect of nimodipine at doses of 60 mgs every 6 hours was found [27]. Why we think our results is different from that of Meyer et al? First of all they included about $25 \%$ of patients with symptomatic generalized epilepsy with mental retardation, these Epilepsies probably increased the mean of crisis per month of the patients studied, with the fact that it is symptomatic generalized epilepsy is known to have

\begin{tabular}{|c|c|}
\hline \multicolumn{2}{|c|}{ Blood pressure/heart (rate[mean \pm SD (range)]) } \\
\hline \multicolumn{2}{|l|}{ Heart rate (beats per minute) $¥$} \\
\hline Before treatment & $79.5 \pm 11.7(69-91)$ \\
\hline One month after treatment & $80 \pm 8.7(72-88)$ \\
\hline Two month after treatment & $78.5 \pm 9.4(69-87)$ \\
\hline three month after treatment & $78.2 \pm 6.7(70-85)$ \\
\hline \multicolumn{2}{|c|}{ Systolic blood pressure $(\mathrm{mm}$ of $\mathrm{Hg}) \neq$} \\
\hline Before treatment & $119 \pm 9.2(104-128)$ \\
\hline One month after treatment & $117 \pm 9.3(108-126)$ \\
\hline Two month after treatment & $116 \pm 9.6(107-125)$ \\
\hline three month after treatment & $116 \pm 10.3(108-126)$ \\
\hline \multicolumn{2}{|c|}{ Diastolic blood pressure ( $\mathrm{mm}$ of $\mathrm{Hg}) @$} \\
\hline Before treatment & $76 \pm 9.6(64-85)$ \\
\hline One month after treatment & $73 \pm 8.7(64-81)$ \\
\hline Two month after treatment & $73 \pm 9.8(62-82)$ \\
\hline three month after treatment & $73 \pm 9.0(64-82)$ \\
\hline
\end{tabular}

$¥$ means Friedman ANOVA and Kendall Coeff. of Concordance: ANOVA Chi Sqr $(\mathrm{N}=26, \mathrm{df}=3)=16.22 \mathrm{p}=0.00097$. Coeff. of Concordance=-0.45 Aver. rank $r=-0.45$ $\neq$ means Friedman ANOVA and Kendall Coeff. of Concordance: ANOVA Chi Sqr. $(\mathrm{N}=26, \mathrm{df}=3)=11.3 \mathrm{p}=0.001$. Coeff. of Concordance=-0.29 Aver. rank $r=-0.27$ @ means Friedman ANOVA and Kendall Coeff. of Concordance: ANOVA Chi Sqr. $(\mathrm{N}=26, \mathrm{df}=3)=12.1 \mathrm{p}=0$.002. Coeff. of Concordance=-0. 28 Aver. rank $r=-0.26$

Table 6: Variability of blood pressure and heart rate initially and during the followed-up. 
Citation: Machado RA, Romero EB, Astencio AMG, Santos AS, Cuartas VB, et al. (2013) Prospective Randomized Controlled Trial of Nimodipine as Add-On Therapy in the Treatment of Focal Refractory Epilepsy Patients: A Pilot Study. J Neurol Neurophysiol 4: 151. doi:10.4172/21559562.1000151

multiple daily crisis. If nimodipine was not effective to control seizures in the generalized epilepsies, its efficacy could be affected comparing the total number of seizures after and before treatment as the author done.

In the present study we decided to select two doses of nimodipine because neurologists are familiar with those doses to treat vasospasm due to subarachnoid hemorrhage, and lower doses have not probably therapeutic effects as Larkin et al. had reported previously [21]. Doses selected by Larkin et al. were lower and as we have mentioned before, did not take into consideration body weight as we did; which could be the cause of failure to find any positive effect of nimodipine in their research. This doses selection bias could affect the evaluation of efficacy in that study. The dependent dose effect found in the present study, favoring the higher doses of nimodipine, support this hypothesis. We did not consider employing doses greater than $3 \mathrm{mg} / \mathrm{kg} /$ day, because neurologists did not have experience with superior doses and the possibility of adverse effects and lack of compliance could be increased.

In spade of previously mentioned explanation, another possible criticism of this study design, is that purposely selecting a low dose outside the therapeutic range, or even a dose that is thought to be marginally effective, raises the same ethical concerns as the utilization of a placebo, but this is not applicable to our study because only a modest reduction of antiepileptic drugs was performed when signs of intoxication were diagnosed.

Fixed-dose studies using multiple doses have gained popularity for evaluating dose-response relationships. While this study design yields valuable information, it does not necessarily reflect the way a drug is likely to be used in clinical practice. To optimize therapeutic benefit during clinical management of a patient, the drug dose is normally titrated within a defined range based on observed response (benefit and tolerability). Dose-titration studies where dose is individualized in this manner, should be recommended to better approximate actual clinical practice $[26,27]$.

Due to the fact that epilepsy has spontaneous remission for months or years, one methodological problem can be to analyze the efficacy of add-on therapy in a short period of time (12-weeks). This problem was mitigated by including only patients with seizures rate greater than five per month. In this population, the likelihood of finding spontaneous seizure remission during the follow-up period is very low.

As nimodipine is a calcium channel blocker and known Pgp inhibitor, it may act facilitating the brain penetration and/or blocking the hepatic clearance (by the Pgp in biliar canaliculus lumen) of AEDs, our patients were simultaneously receiving. At least in animal models, inhibition of Pgp by verapamil $5(\mathrm{mM})$ has been shown to increase significantly the concentration of $\mathrm{PB}$ in extracellular fluid of the cerebral cortex $[8,28]$. We did not discard an effect mediated by antagonism of the calcium channel as associated mechanism. Nevertheless, although other possible bias in our study is that the measurement of the plasma level of all used AEDs in the trial could not be done because only carbamazepine plasma levels can be measured in our laboratories, the increment in plasma level of carbamazepine documented in some of the evaluated patients, suggested that the increased bioavailability of AEDs would be an explanation of pharmacorresistance improvement. Alas, the hypothesis of an increased brain penetration cannot be directly demonstrated, maximum because levels of AEDs in cerebrospinal fluid were not measured.

The synergic effect of nimodipine with the AEDs, patients were receiving, could be other cause for seizures reduction. Before nimodipine administration patients were taking carbamazepine, valproate, benzodiazepines, phenytoin, lamotrigine and topiramate (doses and number of patients under treatment with them are specified in (Table 1), all of them with those AEDs in different combinations. As it's known phenytoin, lamotrigine and carbamazepine all bind in the inner pore of the sodium channel prolonging the 'refractory period' and so inhibiting sustained repetitive firing. Valproate also seems to inhibit rapid repetitive firing but acts at a different site from that at which carbamazepine, lamotrigine and phenytoin act; it also has effects on potentiation of GABAergic inhibition and attenuation of glutamatergic excitation. Benzodiazepines may inhibit the sodium channel at high concentrations which are not usual in clinical practice; they are specific modulators of $\mathrm{GABA}_{\mathrm{A}}$ receptors and act at $\mathrm{GABA}_{\mathrm{A}}$ receptors that contain an $\alpha 1, \alpha 2, \alpha 3$ or $\alpha 5$ - subunit in combination with a $\gamma$-subunit. Topiramate also have actions on sodium channels, but the exact nature and importance of which are unclear, inhibits high-voltage calcium channels and carbonic anhydrase and at high concentrations acts at AMPA/kainate receptors reducing the excitatory actions of glutamate. Have been proposed that lamotrigine and topiramate inhibit $\mathrm{N}$-type calcium channels; lamotrigine may also inhibit P-type channels. However, L-type calcium channels are typically blocked by dihydropyridines (e.g. nimodipine). Calcium entry through L-type channels is the major contributor of calcium to trigger the afterhyperpolarization in certain neuronal subtypes, particularly in the hippocampus, and the somatic expression of L-type channels means that they are ideally placed to open during the depolarization that occurs with an action potential [29]. This mechanism of action of nimodipine is completely different of those of AEDs patients were receiving, alas demonstrating a possible synergistic effect.

Otherwise, it's already known that blockade of L-type calcium channels has a variety of effects on epileptic discharges, and can have both anticonvulsant and proconvulsant effects, possibly by inhibiting synaptic potentiation, yet also inhibiting afterhyperpolarization [29]. Thereby, the antiseizure effect of nimodipine is also another explanation for our results.

Irrespective of the mechanism involved, we consider our results justified further research on the relation between calcium channel blockers, calcium channels and epilepsy.

\section{Conclusion}

Nimodipine, as adjuvant therapy, improves quality of life and seizures rate in patients with refractory focal epilepsy, with a dependentdose effect.

\section{References}

1. Wiebe S (2006) Burden of intractable epilepsy. Adv Neurol 97: 1-4.

2. Schmidt D, Löscher W (2005) Drug resistance in epilepsy: putative neurobiologic and clinical mechanisms. Epilepsia 46: 858-877.

3. Kwan P, Brodie MJ (2000) Early identification of refractory epilepsy. N Engl J Med 342: 314-319.

4. Kwan P, Brodie MJ (2006) Combination therapy in epilepsy: when and what to use. Drugs 66: 1817-1829.

5. Löscher W, Schmidt D (2011) Modern antiepileptic drug development has failed to deliver: ways out of the current dilemma. Epilepsia 52: 657-678.

6. Jacobs MP, Leblanc GG, Brooks-Kayal A, Jensen FE, Lowenstein DH, et al (2009) Curing epilepsy: progress and future directions. Epilepsy Behav 14: 438-445.

7. Löscher W, Klotz U, Zimprich F, Schmidt D (2009) The clinical impact of pharmacogenetics on the treatment of epilepsy. Epilepsia 50: 1-23. 
Citation: Machado RA, Romero EB, Astencio AMG, Santos AS, Cuartas VB, et al. (2013) Prospective Randomized Controlled Trial of Nimodipine as Add-On Therapy in the Treatment of Focal Refractory Epilepsy Patients: A Pilot Study. J Neurol Neurophysiol 4: 151. doi:10.4172/21559562.1000151

Page 8 of 8

8. Potschka H, Fedrowitz M, Löscher W (2002) P-Glycoprotein-mediated efflux of phenobarbital, lamotrigine, and felbamate at the blood-brain barrier: evidence from microdialysis experiments in rats. Neurosci Lett 327: 173-176.

9. Dombrowski SM, Desai SY, Marroni M, Cucullo L, Goodrich K, et al. (2001) Overexpression of multiple drug resistance genes in endothelial cells from patients with refractory epilepsy. Epilepsia 42: 1501-1506.

10. Potschka H, Fedrowitz M, Löscher W (2001) P-glycoprotein and multidrug resistance-associated protein are involved in the regulation of extracellular levels of the major antiepileptic drug carbamazepine in the brain. Neuroreport 12: $3557-3560$.

11. Fortuna A, Alves G, Falcão A, Soares-da-Silva P (2012) Evaluation of the permeability and P-glycoprotein efflux of carbamazepine and several derivatives across mouse small intestine by the Ussing chamber technique. Epilepsia 53: 529-538.

12. Aronica E, Gorter JA, Jansen GH, van Veelen CW, Van Rijen PC, et al. (2013) Expression and cellular distribution of multidrug transporter proteins in two major causes of medically intractable epilepsy: focal cortical dysplasia and glioneuronal tumors. Neuroscience 118: 417-429.

13. Potschka $H$ (2010) Targeting regulation of $A B C$ efflux transporters in brain diseases: a novel therapeutic approach. Pharmacol Ther 125: 118-127.

14. Baltes S, Gastens AM, Fedrowitz M, Potschka H, Kaever V, et al. (2007) Differences in the transport of the antiepileptic drugs phenytoin, levetiracetam and carbamazepine by human and mouse P-glycoprotein. Neuropharmacology 52: $333-346$.

15. Weiss J, Kerpen CJ, Lindenmaier H, Dormann SM, Haefeli WE (2003) Interaction of antiepileptic drugs with human P-glycoprotein in vitro. J Pharmacol Exp Ther 307: 262-267.

16. Fisher GA, Lum BL, Hausdorff J, Sikic BI (1996) Pharmacological considerations in the modulation of multidrug resistance. Eur J Cancer 32A: 1082-1088.

17. Perucca E (1982) Pharmacokinetic interactions with antiepileptic drugs. Clin Pharmacokinet 7: 57-84
18. Patsalos PN, Fröscher W, Pisani F, van Rijn CM (2002) The importance of drug interactions in epilepsy therapy. Epilepsia 43: 365-385

19. Langley MS, Sorkin EM (1989) Nimodipine. A review of its pharmacodynamic and pharmacokinetic properties, and therapeutic potential in cerebrovascular disease. Drugs 37: 669-699.

20. Allen GS, Ahn HS, Preziosi TJ, Battye R, Boone SC, et al. (1983) Cerebra arterial spasm--a controlled trial of nimodipine in patients with subarachnoid hemorrhage. N Engl J Med 308: 619-624.

21. Larkin JG, McKee PJ, Blacklaw J, Thompson GG, Morgan IC, et al. (1991) Nimodipine in refractory epilepsy: a placebo-controlled, add-on study. Epilepsy Res 9: 71-77.

22. Kwan P, Brodie MJ (2005) Potential role of drug transporters in the pathogenesis of medically intractable epilepsy. Epilepsia 46: 224-235.

23. Löscher W (2005) Mechanisms of drug resistance. Epileptic Disord 7 Supp 1: S3-9

24. Löscher W, Potschka H (2005) Blood-brain barrier active efflux transporters: ATP-binding cassette gene family. NeuroRx 2: 86-98.

25. Löscher W, Klotz U, Zimprich F, Schmidt D (2009) The clinical impact of pharmacogenetics on the treatment of epilepsy. Epilepsia 50: 1-23.

26. lannetti P, Spalice A, Parisi P (2005) Calcium-channel blocker verapami administration in prolonged and refractory status epilepticus. Epilepsia 46: 967 969

27. Meyer FB, Cascino GD, Whisnant JP, Sharbrough FW, Ivnik RJ, et al. (1995) Nimodipine as an add-on therapy for intractable epilepsy. Mayo Clin Proc 70: 623-627.

28. Cereghino JJ (1992) Clinical trial design for antiepileptic drugs. Ann Neurol 32 393-394.

29. Walker MC, Surges R, Fisher A (2009) Mechanisms of Antiepileptic Drug Action. In: Shorvon S, Perucca E, Engel J Jr. eds. The Treatment of Epilepsy. Third edition. West Sussex: John Wiley \& Sons, Ltd. 91-108. 